\title{
Chebyshev wavelets collocation method for simulating a two-phase flow of immiscible fluids in a reservoir with different capillary effects
}

\author{
Gaston Edem Awashie ${ }^{1}$ Peter Amoako-Yirenkyi ${ }^{1,2}$ (I) Isaac Kwame Dontwi ${ }^{1,2}$
}

Received: 9 June 2018 / Accepted: 13 December 2018 / Published online: 30 January 2019

(c) The Author(s) 2019

\begin{abstract}
At least in the last 10 years, considerable effort has been given to studying the dynamics of fluid flow in porous media. The phenomena is widely applicable in many areas of science and engineering. In many cases, the effect of capillary pressure and discontinuities in the two-phase flow dynamics is not fully clear, especially in petroleum reservoirs. In this paper, we introduce a new method based on the Chebyshev wavelets collocation method and the so-called operational matrices of integration. The method was implemented specifically for an oil-water-phase flow in heterogeneous reservoir using different capillary pressure treatments. Convergence and accuracy of this method were established and used to simulate the partial differential equations governing the two-phase model. The method incorporates the various conditions of the complex governing equations as a single system. The system is subsequently reduced into a simple set of algebraic equations making the problem easier to solve. Numerical results showed that the method is able to account for the expected discontinuities occurring in the flow process. It was also found that these discontinuities or jumps in the two-phase flow are caused by the capillary pressure as expected physically.
\end{abstract}

Keywords Two-phase flow $\cdot$ Chebyshev wavelets $\cdot$ Operational matrices

\section{Introduction}

In the past decade, considerable effort has been made in studying the dynamics of fluid flow in porous media due to its applicability in many areas of science and engineering (Soulaine et al. 2013; Pasquier et al. 2017; Ahammad and Alam 2017), like hydrology, ground water remediation, membrane separation, polymer filtration, and oil and gas recovery from reservoirs (Epshteyn and Riviere 2006; Peaceman 1977; Zhong et al. 2013; Al-Rbeawi 2017). In petroleum reservoirs, the process is studied to predict their future performance and also optimize recovery processes in the reservoirs (Ahammad and Alam 2017; Begum 2009). This has led to the modification and proposition of models and methods for numerical reservoir simulation. According to Ewing (1983), reservoir simulation is a standard tool for

Peter Amoako-Yirenkyi

amoakoyirenkyi@knust.edu.gh

1 Department of Mathematics, Kwame Nkrumah University of Science and Technology, Kumasi, Ghana

2 Center for Scientific Computing and Industrial Modeling, National Institute for Mathematical Sciences, Kumasi, Ghana predicting the flow of fluids through porous media under various operating conditions.

Oil industries, for instance, are interested in improving numerical methods to simulate the recovery of oil to exploit the reservoirs in an optimal way (Pasquier et al. 2017). Fluids such as water or gas are mostly injected into the oil reservoir to improve the recovery of oil from the medium, changing the dynamics of the flow in the reservoir. Detailed study of fluid flow in porous media is challenging even in the case of the single-phase flow (Mozolevski and Schuh 2013). Flow simulation in reservoirs has been studied using finite-difference methods, finite-element methods, and finitevolume methods among others over the past years (El-Amin et al. 2015; Foroozesh et al. 2008; Sun and Yuan 2015; Yuan et al. 2015). The challenge is that these methods are either chosen based on the nature of the problem and the problem dynamics, or in some cases, the methods for simulation are changed anytime the dynamics of the process changes. Particularly, in heterogeneous porous media, most methods cannot clearly capture the expected reservoir properties due to the consistent changing dynamics in heterogeneous media. It appears that methods that can adapt to such changes are 
well suited to solve flow problems in heterogeneous porous media.

The two-phase flow in a reservoir has been investigated by some authors (Amaziane and Jurak 2007; Hoteit and Firoozabadi 2008b; Zhong et al. 2013; Pasquier et al. 2017). The models describing flow of fluids in a reservoir are mostly nonlinear or coupled partial differential equations with its solution providing insight into the dynamics of the flow process. The simultaneous flow of fluids in a reservoir and many other porous media is a highly complex phenomena (Alam 2017; Szymkiewicz 2007). Numerical simulation of two-phase flow through a reservoir remains very challenging (Ahammad and Alam 2017; de la Cruz and Monsivais 2013). The effect of capillary pressure, permeabilities and heterogeneity significantly influences the flow path of the fluids (Kou and Sun 2010; El-Amin et al. 2015).

Wavelets have numerous applications and have been extensively used for numerical approximations in relevant literature over the past few decades. it is important to note that wavelet methods have gained great popularity for finding numerical solutions to many forms of differential and integral equations (Araghi et al. 2012; Epshteyn and Riviere 2006; Heydari et al. 2012; Hooshmandasl et al. 2012; Lepik 2011; Li 2010; Alam 2017) due to its high level of accuracy, speed, and efficiency in estimating the solutions. Different wavelet families were applied in various studies examples of which are Haar, Daubechies, Chebyshev, Legendre, and B-spline wavelets. The hallmark of wavelets is their ability to study the function at different scale features (Daubechies 1992).

Chebyshev wavelets are the types of wavelets constructed from Chebyshev polynomials as their basis functions. They have very excellent interpolating properties and gives better accuracy for numerical approximations (Heydari et al. 2014). Our purpose in this paper is to propose the Chebyshev wavelet collocation method for computing oil-water two-phase fluid flow in a reservoir. The present work is a continuation of the earlier work of same authors, AmoakoYirenkyi et al. (2016) in which the Chebyshev wavelet collocation method was used for the numerical simulation of the single-phase flow in a reservoir.

In this paper, Chebyshev wavelet collocation method is used mutually with the operational matrix of integration to simulate the two-phase flow process in a reservoir with different capillary pressures. This paper is outlined as follows: in "Two-phase flow model", the two-phase flow model is reviewed. In "First kind Chebyshev wavelets and its properties", we describe the Chebyshev wavelets and its properties as the solution scheme. In "Chebyshev wavelet formulation of two-phase flow model", we present the wavelet formulation of the two-phase flow model. The approximate solution to the flow problem is discussed in "Numerical results". Finally, we conclude in "Conclusions".

\section{Two-phase flow model}

The two-phase flow of fluids in a reservoir is governed by nonlinear or coupled partial differential equation. This flow model consists of the conservation of mass equations and the Darcy's law (Peaceman 1977). The conservation of mass equation is given by Epshteyn and Riviere (2006) and Peace$\operatorname{man}(1977)$

$\frac{\partial}{\partial t}\left(\phi S_{\alpha}\right)+\nabla \cdot u_{\alpha}=q_{\alpha}, \quad \alpha=w, o$,

where $\alpha$ represents the fluid phase (water being the wetting phase denoted by $w$ and oil, the non-wetting phase denoted by $o$ ), $\phi$ is the porosity, $q_{\alpha}$ is the injection or production rate per unit volume at phase $\alpha(1 / \mathrm{s}), S_{\alpha}$ is the saturation at phase $\alpha$, and $u_{\alpha}$ represents the Darcy's velocity $(\mathrm{m} / \mathrm{s})$. The Darcy's law defines a linear relationship between the velocity of the fluid and the gradient of phase pressure $P_{\alpha}(\mathrm{Pa})$ :

$u_{\alpha}=-K \frac{k_{r \alpha}}{\mu_{\alpha}}\left(\nabla P_{\alpha}+\rho_{\alpha} g \nabla Z\right), \quad \alpha=w, o$,

where $K$ is the absolute permeability, $\left(\mathrm{m}^{2}\right), k_{r \alpha}$ is the relative permeability (-) of phase $\alpha, \rho_{\alpha}\left(\mathrm{kg} / \mathrm{m}^{3}\right)$, and $\mu_{\alpha},(\mathrm{Pas})$ are density and viscosity of phase $\alpha$, respectively. Water and oil are assumed to fill the entire pore space in the medium. That is

$S_{\mathrm{o}}+S_{\mathrm{w}}=1$.

The pressure in the non-wetting fluid is mostly higher than the pressure of the wetting fluid and the pressure difference gives the capillary pressure $P_{\mathrm{c}}$ which is a function of saturation (Peaceman 1977):

$P_{\mathrm{c}}\left(S_{\mathrm{w}}\right)=P_{\mathrm{o}}-P_{\mathrm{w}}$.

The coupled equations are decoupled into pressure-saturation equations which are solved simultaneously for the model. Substituting Eq. (2) into Eq. (1) and expanding in terms of the wetting and non-wetting phases, we have

$\frac{\partial}{\partial t}\left(\phi S_{\mathrm{w}}\right)+\nabla \cdot\left(-K \frac{k_{\mathrm{rw}}}{\mu_{\mathrm{w}}}\left(\nabla P_{\mathrm{w}}+\rho_{\mathrm{w}} g \nabla Z\right)\right)=q_{\mathrm{w}}$,

$\frac{\partial}{\partial t}\left(\phi S_{\mathrm{o}}\right)+\nabla \cdot\left(-K \frac{k_{\mathrm{ro}}}{\mu_{\mathrm{o}}}\left(\nabla P_{\mathrm{o}}+\rho_{\mathrm{o}} g \nabla Z\right)\right)=q_{\mathrm{o}}$.

The constraints from Eqs. (3) and (4) give the saturation of oil to be $S_{\mathrm{o}}=1-S_{\mathrm{w}}$ and pressure of water to be $P_{\mathrm{w}}=P_{\mathrm{o}}-P_{\mathrm{c}}$ are then substituted into Eqs. (5) and (6). The resulting expressions are

$\frac{\partial}{\partial t}\left(\phi S_{\mathrm{w}}\right)+\nabla \cdot\left(-K \frac{k_{\mathrm{rw}}}{\mu_{\mathrm{w}}}\left(\nabla\left(P_{\mathrm{o}}-P_{\mathrm{c}}\right)+\rho_{\mathrm{w}} g \nabla Z\right)\right)=q_{\mathrm{w}}$, 
$\frac{\partial}{\partial t} \phi\left(1-S_{\mathrm{w}}\right)+\nabla \cdot\left(-K \frac{k_{\mathrm{ro}}}{\mu_{\mathrm{o}}}\left(\nabla P_{\mathrm{o}}+\rho_{\mathrm{o}} g \nabla Z\right)\right)=q_{\mathrm{o}}$.

Based on the assumption that the porosity is constant, the pressure equation was obtained from Eqs. (7) and (8) as

$$
\begin{aligned}
\nabla & \cdot\left(-K\left(\frac{k_{\mathrm{rw}}}{\mu_{\mathrm{w}}}+\frac{k_{\mathrm{ro}}}{\mu_{\mathrm{o}}}\right) \nabla P_{\mathrm{o}}\right) \\
& +\nabla \cdot\left(-K g\left(\frac{k_{\mathrm{rw}}}{\mu_{\mathrm{w}}} \rho_{\mathrm{w}}+\frac{k_{\mathrm{ro}}}{\mu_{\mathrm{o}}} \rho_{\mathrm{o}}\right) \nabla Z\right) \\
& +\nabla \cdot\left(K \frac{k_{\mathrm{rw}}}{\mu_{\mathrm{w}}} \nabla P_{\mathrm{c}}\right)=q_{\mathrm{w}}+q_{\mathrm{o}} .
\end{aligned}
$$

Let $\lambda_{\alpha}=k_{r \alpha} / \mu_{\alpha}$ be the mobility of phase $\alpha$, then Eq. (9) becomes

$$
\begin{aligned}
& \nabla \cdot\left(-K\left(\lambda_{\mathrm{w}}+\lambda_{\mathrm{o}}\right) \nabla P_{\mathrm{o}}\right)+\nabla \cdot\left(-K g\left(\lambda_{\mathrm{w}} \rho_{\mathrm{w}}+\lambda_{\mathrm{o}} \rho_{\mathrm{o}}\right) \nabla Z\right) \\
& \quad+\nabla \cdot\left(K \lambda_{\mathrm{w}} \nabla P_{\mathrm{c}}\right)=q_{\mathrm{w}}+q_{\mathrm{o}} .
\end{aligned}
$$

This equation is solved for the pressure of oil phase which can be used in estimating the pressure of water from Eq. (4). The Darcy velocity for water is then computed from the pressure distribution. The saturation equation is given as

$\frac{\partial}{\partial t}\left(\phi S_{\mathrm{w}}\right)+\nabla \cdot u_{\mathrm{w}}=q_{\mathrm{w}}$.

That is, the pressure-saturation equations governing twophase flow in reservoirs are given by Eqs. (10) and (11), respectively. Let the boundary $\partial \Omega$ of the computational domain $\Omega$ be decomposed into the Dirichlet part $\Gamma^{\mathrm{D}}$ and Neumann part $\Gamma^{\mathrm{N}}$, where $\partial \Omega=\Gamma^{\mathrm{D}} \bigcup \Gamma^{\mathrm{N}}$ and $\Gamma^{\mathrm{D}} \bigcap \Gamma^{\mathrm{N}}=\{\}$. The boundary conditions associated with the governing equations (10) and (11) are given as

$P_{\mathrm{o}} \quad\left(\right.$ or $\left.P_{\mathrm{w}}\right)=P^{\mathrm{D}} \quad$ on $\Gamma^{\mathrm{D}}$,

$u_{\alpha} \cdot n=u_{\alpha}^{\mathrm{N}} \quad$ on $\Gamma^{\mathrm{N}}$,

$S_{\mathrm{w}}=S^{\mathrm{N}}$ on $\Gamma^{\mathrm{N}}$,

and the initial saturation of the non-wetting phase is given by

$S_{\mathrm{w}}=S_{\mathrm{w}}^{0}$.

\section{First kind Chebyshev wavelets and its properties}

Wavelets are a class of functions constructed from a dilation and translation of a single function called the mother wavelet. If both dilation parameter, $a$, and translation parameter, $b$, are allowed to vary continuously, we obtain a family of continuous wavelets as

$\psi_{a, b}(t)=|a|^{-\frac{1}{2}} \psi\left(\frac{t-b}{a}\right), \quad a, b \in \mathbb{R}, a \neq 0$.
Restricting the parameters $a$ and $b$ to assume discrete values as $a=a_{0}^{-n}$ and $b=m b_{0} a_{0}^{-n}$, where $a_{0}>1, b_{0}>0$, with $n$ and $m$ being positive integers, gives a family of discrete wavelets

$\psi_{n, m}(t)=\left|a_{0}\right|^{\frac{n}{2}} \psi\left(a_{0}^{n} t-m b_{0}\right), \quad n, m \in \mathbb{Z}$

which form a wavelets basis in $L^{2}(\mathbb{R})$. These basis are orthonormal basis of the function space when $a_{0}=2$ and $b_{0}=1$.

For any positive integer $k$, the first kind Chebyshev wavelets family is defined on the interval $[0,1]$ as

$\psi_{n, m}(t)= \begin{cases}2^{k / 2} \mathcal{T}_{m}\left(2^{k} t-2 n+1\right), & \frac{n-1}{2^{k-1}} \leq t \leq \frac{n}{2^{k-1}} \\ 0, & \text { otherwise }\end{cases}$

constructed as a function of four arguments $\left(\psi_{n, m}(t)=\psi(n, m, k, t)\right)$, where $n=1,2, \ldots, 2^{k-1}, m$ is the degree of the first kind Chebyshev polynomials, $t$ is the normalized time and

$\mathcal{T}_{m}(t)= \begin{cases}\frac{1}{\sqrt{\pi}}, & m=0 \\ \sqrt{\frac{2}{\pi}} T_{m}(t), & m>0 .\end{cases}$

Here, $T_{m}(t)$ are the Chebyshev polynomials of order $m$ defined on the interval $[-1,1]$. The coefficients in Eq. (19) are to ensure orthonormality of the constructed wavelets. The Chebyshev polynomials are generally calculated recursively from the set of equations:

$T_{0}(t)=1, \quad T_{1}(t)=t$,

$T_{m}(t)=2 t T_{m-1}-T_{m-2} ; \quad m=2,3, \ldots, M-1$.

where $M$ is a fixed positive integer greater than 2 . The set of Chebyshev Polynomials are orthogonal with respect to the weight function (Heydari et al. 2014):

$w(t)=\frac{1}{\sqrt{1-t^{2}}}$.

To ensure orthogonality in dealing with the Chebyshev wavelets, the weight function $w(t)$ must be dilated and translated (Araghi et al. 2012; Heydari et al. 2014) as

$w_{n}(t)=w\left(2^{k} t-2 n+1\right)$.

Any arbitrary function $f(t)$ defined on the interval $[0,1)$ which is squared integrable can be expanded by the Chebyshev wavelets family as

$f(t)=\sum_{n=1}^{\infty} \sum_{m=0}^{\infty} c_{n m} \psi_{n m}(t)$ 
where $c_{n m}=\left\langle f(t), \psi_{n m}(t)\right\rangle_{L_{\mathrm{w}}^{2}[0,1)}$ and $\langle,\rangle_{L_{\mathrm{w}}^{2}[0,1)}$ represents the inner product in $L^{2}[0,1)$ with respect to weight function, defined as

$c_{n m}=\left\langle f(t), \psi_{n m}(t)\right\rangle_{L_{\mathrm{w}}^{2}[0,1)}=\int_{0}^{1} f(t) \psi_{n m}(t) w_{n}(t) \mathrm{d} t$.

For approximations with the Chebyshev wavelets, the above series may be truncated as

$f(t) \cong \sum_{n=1}^{2^{k-1}} \sum_{m=0}^{M-1} c_{n m} \psi_{n m}(t)=C^{\mathrm{T}} \Psi(t)$

where $C, \Psi(t)$ are $2^{k-1} M$ column vectors given by

$$
\begin{aligned}
C= & {\left[c_{1,0}, c_{1,1}, \ldots, c_{1, M-1}, c_{2,0}, c_{2,1} \cdots, c_{2, M-1}, \ldots, c_{2^{k-1}, 0},\right.} \\
& \left.c_{2^{k-1}, 1}, \ldots, c_{2^{k-1} M-1}\right]^{\mathrm{T}}
\end{aligned}
$$

$\Psi(t)=\left[\psi_{1,0}(t), \psi_{1,1}(t), \ldots, \psi_{1, M-1}, \psi_{2,0}, \psi_{2,1} \cdots, \psi_{2 M-1}(t), \ldots\right.$,

$$
\left.\psi_{2^{k-1} 0}(t), \ldots, \psi_{2^{k-1} M-1}(t)\right]^{\mathrm{T}} .
$$

Likewise, a two variable function $f(x, t)$ defined on the square $[0,1) \times[0,1)$ may be expanded using the Chebyshev wavelets basis as

$f(x, t)=\sum_{n=1}^{\infty} \sum_{n^{*}=1}^{\infty} \sum_{m=0}^{\infty} \sum_{m^{*}=0}^{\infty} d_{n, n^{*}, m, m^{*}} \psi_{n, m}(x) \psi_{n^{*}, m^{*}}(t)$,

where $d_{n, n^{*}, m, m^{*}}=\left\langle f(x, t), \psi_{n, m}(x) \psi_{n^{*}, m^{*}}(t)\right\rangle_{L_{\mathrm{w}}^{2}([0,1) \times[0,1))}$. If the infinite series representation of $f(x, t)$ in Eq. (29) is truncated, then we have

$f(x, t) \cong \sum_{n=1}^{2^{k-1}} \sum_{n^{*}=1}^{2^{k-1}} \sum_{m=0}^{M-1} \sum_{m^{*}=0}^{M-1} d_{n, n^{*}, m, m^{*}} \psi_{n, m}(x) \psi_{n^{*}, m^{*}}(t)$,

$=\sum_{n=1}^{2^{k-1}} \sum_{m=0}^{M-1} \psi_{n, m}(x)\left(\sum_{n^{*}=1}^{2^{k-1}} \sum_{m^{*}=0}^{M-1} d_{n, n^{*}, m, m^{*}} \psi_{n^{*}, m^{*}}(t)\right)$

$=\sum_{n=1}^{2^{k-1}} \sum_{m=0}^{M-1} \psi_{n, m}(x) D_{n m}^{\mathrm{T}} \Psi(t)$

where $D_{n m}$ and $\Psi(t)$ are $2^{k-1} M$ column vectors which can be seen as

$$
\begin{aligned}
D_{n m}= & {\left[d_{n, 1, m, 0}, d_{n, 1, m, 1}, \ldots, d_{n, 1, m, M-1}, \ldots, d_{n, 2^{k-1}, m, 0},\right.} \\
& \left.d_{n, 2^{k-1}, m, 1}, \ldots, d_{n, 2^{k-1} m, M-1}\right]^{\mathrm{T}}
\end{aligned}
$$

$$
\begin{gathered}
\Psi(t)=\left[\psi_{1,0}(t), \psi_{1,1}(t), \ldots, \psi_{1, M-1}, \psi_{2,0}, \psi_{2,1} \cdots, \psi_{2 M-1}(t), \ldots,\right. \\
\left.\psi_{2^{k-1} 0}(t), \ldots, \psi_{2^{k-1} M-1}(t)\right]^{\mathrm{T}} .
\end{gathered}
$$

In like manner, we can write Eq. (34) as

$$
f(x, t) \cong \sum_{n=1}^{2^{k-1}} \sum_{m=0}^{M-1} \psi_{n, m}(x) D_{n m}^{\mathrm{T}} \Psi(t)=\Psi^{\mathrm{T}}(x) D \Psi(t),
$$

where $D$ is a $2^{k-1} M \times 2^{k-1} M$ matrix.

\section{Convergence analysis}

Let

$\psi_{n, m}(t)=\left|a_{0}\right|^{\frac{n}{2}} \psi\left(a_{0}^{n} t-m b_{0}\right), \quad n, m \in \mathbb{Z}$,

where $\psi_{n, m}(t)$ form the wavelet basis of $L^{2}(\mathbb{R})$. Interesting to note, when $a_{0}=2$ and $b_{0}=1, \psi_{n, m}(t)$ forms an orthonormal basis for $L^{2}(\mathbb{R})$. Theorems 3.1 and 3.2 give the convergence of the first kind Chebyshev wavelets expansion of a function $f(t)$.

Theorem 3.1 If the Chebyshev wavelet expansion of a continuous function $f(t)$ converges uniformly, then the Chebyshev wavelet expansion converges to the function $f(t)$ (Adibi and Assari 2010).

Proof Let

$g(t)=\sum_{n=1}^{\infty} \sum_{m=0}^{\infty} c_{n m} \psi_{n m}(t)$

where $c_{n m}=\left\langle f(t), \psi_{n m}(t)\right\rangle_{w_{k}}$. When both sides of Eq. (37) by $\psi_{p q}(t) w_{k}(t)$, we have

$$
\begin{aligned}
\int_{0}^{1} g(t) \psi_{p q}(t) w_{k}(t) \mathrm{d} t \\
\quad=\int_{0}^{1} \sum_{n=1}^{\infty} \sum_{m=0}^{\infty} c_{n m} \psi_{n m}(t) \psi_{p q}(t) w_{k}(t) \mathrm{d} t
\end{aligned}
$$

where $p$ and $q$ are fixed and evaluating the integral term wise which is justified by uniform convergence on $[0,1]$

$$
\begin{aligned}
& \int_{0}^{1} g(t) \psi_{p q}(t) w_{k}(t) \mathrm{d} t \\
& \quad=\sum_{n=1}^{\infty} \sum_{m=0}^{\infty} c_{n m} \int_{0}^{1} \psi_{n m}(t) \psi_{p q}(t) w_{k}(t) \mathrm{d} t
\end{aligned}
$$


$=c_{p q}$.

Thus, $\left\langle g(t), \psi_{n m}(t)\right\rangle_{w_{k}}=c_{n m}$ for $n=1,2, \ldots$ and $m=0,1, \ldots$ This implies by consequence that $f$ and $g$ have the same Fourier expansion based on the Chebyshev wavelets. We, therefore, conclude that $f(t)=g(t)$ for $t \in[0,1]$.

Theorem 3.2 If a function $f(t) \in L^{2}([0,1])$, with bounded second order derivative $\left|f^{\prime \prime}(t)\right| \leq \mathcal{K}$ can be expanded as a sum of infinite Chebyshev wavelets:

$f(t)=\sum_{n=1}^{\infty} \sum_{m=0}^{\infty} c_{n m} \psi_{n m}(t)$

then

$\left|c_{n m}\right| \leq \frac{\sqrt{2 \pi} \mathcal{K}}{(2 n)^{\frac{5}{2}}\left(m^{2}-1\right)}$,

which implies the Chebyshev wavelets expansion converges uniformly to $f(t)$.

Proof It follows from the definition:

$c_{n m}=\left\langle f(t), \psi_{n m}(t)\right\rangle_{w_{k}}$,

that

$$
\begin{aligned}
c_{n m} & =\int_{0}^{1} f(t) \psi_{n m}(t) w_{k}(t) \mathrm{d} t, \\
& =\int_{(n-1) / 2^{k-1}}^{n / 2^{k-1}} 2^{k / 2} f(t) \mathcal{T}_{m}\left(2^{k} t-2 n+1\right) w\left(2^{k} t-2 n+1\right) .
\end{aligned}
$$

Let $\cos (\theta)$ be substituted into Eq. (44) for $2^{k} t-2 n+1$ for $m>1$ yields

$$
c_{n m}=\frac{\sqrt{2}}{2^{k / 2} \sqrt{\pi}} \int_{0}^{\pi} f\left(\frac{\cos (\theta)+2 n-1}{2^{k}}\right) \cos (m \theta) \mathrm{d} \theta .
$$

Using integration by parts, we obtain

$$
\begin{aligned}
c_{n m}= & \left.\frac{\sqrt{2}}{2^{k / 2} \sqrt{\pi}} f\left(\frac{\cos (\theta)+2 n-1}{2^{k}}\right)\left(\frac{\sin (m \theta)}{m}\right)\right|_{0} ^{\pi} \\
& +\frac{\sqrt{2}}{2^{3 k / 2} m \sqrt{2 \pi}} \int_{0}^{\pi} f^{\prime}\left(\frac{\cos (\theta)+2 n-1}{2^{k}}\right) \sin (m \theta) \sin (\theta) \mathrm{d} \theta
\end{aligned}
$$

$$
\begin{aligned}
= & \frac{1}{2^{3 k / 2} m \sqrt{2 \pi}} f^{\prime}\left(\frac{\cos (\theta)+2 n-1}{2^{k}}\right) \\
& \times\left.\left(\frac{\sin (m-1) \theta}{m-1}-\frac{\sin (m+1) \theta}{m+1}\right)\right|_{0} ^{\pi} \\
& +\frac{1}{2^{5 k / 2} m \sqrt{2 \pi}} \int_{0}^{\pi} f^{\prime \prime}\left(\frac{\cos (\theta)+2 n-1}{2^{k}}\right) \sin (\theta) H(\theta) \mathrm{d} \theta,
\end{aligned}
$$

where

$H(\theta)=\frac{\sin (m-1) \theta}{m-1}-\frac{\sin (m+1) \theta}{m+1}$.

Evaluating the above integral, we have

$c_{n m}=\frac{1}{2^{5 k / 2} m \sqrt{2 \pi}} \int_{0}^{\pi} f^{\prime \prime}\left(\frac{\cos (\theta)+2 n-1}{2^{k}}\right) \sin (\theta) H(\theta) \mathrm{d} \theta$.

Taking the magnitude of both sides

$\left|c_{n m}\right|=\left|\frac{1}{2^{5 k / 2} m \sqrt{2 \pi}} \int_{0}^{\pi} f^{\prime \prime}\left(\frac{\cos (\theta)+2 n-1}{2^{k}}\right) \sin (\theta) H(\theta) \mathrm{d} \theta\right|$

$\leq \frac{1}{2^{5 k / 2} m \sqrt{2 \pi}} \int_{0}^{\pi}\left|f^{\prime \prime}\left(\frac{\cos (\theta)+2 n-1}{2^{k}}\right) \sin (\theta) H(\theta)\right| \mathrm{d} \theta$

$\leq \frac{\mathcal{K}}{2^{5 k / 2} m \sqrt{2 \pi}} \int_{0}^{\pi}|\sin (\theta) H(\theta)| \mathrm{d} \theta$.

However

$$
\begin{aligned}
& \int_{0}^{\pi}|\sin (\theta) H(\theta)| \mathrm{d} \theta \\
& \quad=\int_{0}^{\pi}\left|\sin (\theta)\left(\frac{\sin (m-1) \theta}{m-1}-\frac{\sin (m+1) \theta}{m+1}\right)\right| \mathrm{d} \theta \\
& \leq \int_{0}^{\pi}\left|\frac{\sin (\theta) \sin (m-1) \theta}{m-1}\right|+\left|\frac{\sin (\theta) \sin (m+1) \theta}{m+1}\right| \mathrm{d} \theta \\
& \leq \frac{2 m \pi}{\left(m^{2}-1\right)} .
\end{aligned}
$$

Hence 
$\left|c_{n m}\right| \leq \frac{\mathcal{K}}{2^{5 k / 2} m \sqrt{2 \pi}}\left(\frac{2 m \pi}{m^{2}-1}\right)$.

By rationalizing and simplifying the expression to the right of Eq. (56), it can be seen that

$\left|c_{n m}\right| \leq \frac{\sqrt{2 \pi} \mathcal{K}}{2^{5 k / 2}\left(m^{2}-1\right)}$.

Since $n \leq 2^{k-1}$, we have inequality (57) becoming

$\left|c_{n m}\right|<\frac{\sqrt{2 \pi} \mathcal{K}}{(2 n)^{5 / 2}\left(m^{2}-1\right)}$.

Therefore, the wavelets expansion $\sum_{n=1}^{\infty} \sum_{m=0}^{\infty} c_{n m} \psi_{n m}(t)$ converges to $f(t)$ uniformly supported by Theorem 3.1.

\section{Error analysis}

Error function, $E_{t}$, can be computed for a function $f(t)$ approximated by the Chebyshev wavelets series as

$E_{t}=\left|f(t)-\sum_{n=1}^{2^{k-1}} \sum_{m=0}^{M-1} c_{n m} \psi_{n m}\right|$,

where the absolute error values can be obtained for $t=t_{j}$, for every $t_{j} \in[0,1]$ based on Eq. (59).

Assume the Chebyshev wavelets basis has $P$ vanishing moments, then the wavelets coefficients are bounded. That is

$\left|c_{n m}\right| \leq \mathcal{K} 2^{-n(P+1 / 2)} \max _{\xi \in[0,1]}\left|f^{(P)}(\xi)\right|$,

where $\mathcal{K}$ is a constant. Associated error approximations by the Chebyshev wavelets is established in Theorem 3.3.

Theorem 3.3 Suppose $C^{\mathrm{T}} \psi(t)=\sum_{n=1}^{2^{k-1}} \sum_{m=0}^{M-1} c_{n m} \psi_{n m}$ is the Chebyshev wavelets approximation to a function $f(t)$ in the function space, then the error is bounded by the expression:

$\left\|E_{t}\right\| \leq \frac{1}{P ! 2^{p(k-1)}} \max _{\xi \in[0,1]}\left|f^{(P)}(\xi)\right|$.

Proof In the use of the Chebyshev wavelets, the interval $[0,1]$ is divided into $2^{k-1}$ subintervals $I_{n}=\left[\frac{n-1}{2^{k-1}}, \frac{n}{2^{k-1}}\right]$ on which the function $f(t)$ is approximated.

The norm as defined by the inner product space for the function $E_{t}$ is given as

$$
\begin{aligned}
\left\|E_{t}\right\|^{2} & =\left\|f(t)-C^{\mathrm{T}} \psi(t)\right\|^{2} \\
& =\int_{0}^{1} W(t)\left(f(t)-C^{\mathrm{T}} \psi(t)\right)^{2} \mathrm{~d} t \\
& =\sum_{n=1}^{2^{k-1}} \int_{\frac{n-1}{2^{k-1}}}^{\frac{n}{2^{k-1}}} W(t)\left(f(t)-C^{\mathrm{T}} \psi(t)\right)^{2} \mathrm{~d} t \\
& \leq \sum_{n=1}^{\frac{n}{2^{k-1}}} \int_{\frac{n-1}{2^{k-1}}}^{\frac{n}{2^{k-1}}} W(t)\left(f(t)-S_{P}(t)\right)^{2} \mathrm{~d} t,
\end{aligned}
$$

where $S_{P}(t)$ is any polynomial of degree $P$ that interpolates $f(t)$ on the subintervals with the error bound being

$\left|f(t)-S_{P}(t)\right| \leq \frac{1}{P ! 2^{p(k-1)}} \max _{\xi_{n} \in I_{n}}\left|f^{(P)}\left(\xi_{n}\right)\right|$.

Hence

$$
\begin{aligned}
\left\|E_{t}\right\|^{2} & \leq \sum_{n=1}^{2^{k-1}} \int_{\frac{n-1}{2^{k-1}}}^{\frac{n}{2^{k-1}}} W_{n}(t)\left(\frac{1}{P ! 2^{p(k-1)}} \max _{\xi_{n} \in I_{n}}\left|f^{(P)}\left(\xi_{n}\right)\right|\right)^{2} \mathrm{~d} t \\
& \leq \sum_{n=1}^{2^{k-1}} \int_{\frac{n-1}{2^{k-1}}}^{\frac{n}{2^{k-1}}} W_{n}(t)\left(\frac{1}{P ! 2^{p(k-1)}} \max _{\xi \in[0,1]}\left|f^{(P)}(\xi)\right|\right)^{2} \mathrm{~d} t \\
& =\int_{0}^{1} W_{n}(t)\left(\frac{1}{P ! 2^{p(k-1)}} \max _{\xi \in[0,1]}\left|f^{(P)}(\xi)\right|\right)^{2} \mathrm{~d} t \\
& =\left\|\frac{1}{P ! 2^{p(k-1)}} \max _{\xi \in[0,1]}\left|f^{(P)}(\xi)\right|\right\|^{2} .
\end{aligned}
$$

\section{Accuracy analysis}

The evaluation of the accuracy of a numerical method is crucial to describing the performance and applicability to solving problems. Theorem 3.4 shows the accuracy of the Chebyshev wavelets representation of a function in the function space.

Theorem 3.4 Given the second-order derivative squareintegrable function $f(t)$ defined on the interval $[0,1)$ with bounded second-order derivative for some constant $\mathcal{K}$, say $\left|f^{\prime \prime}(t)\right| \leq \mathcal{K}$, then

$\sigma_{k, M}<\frac{\sqrt{\pi} \mathcal{K}}{2^{2}}\left(\sum_{n=2^{k-1}+1}^{\infty} \sum_{m=M}^{\infty} \frac{1}{n^{5}\left(m^{2}-1\right)^{2}}\right)^{\frac{1}{2}}$

where

$\sigma_{k, M}=\left(\int_{0}^{1}\left|f(t)-\sum_{n=1}^{2^{k-1}} \sum_{m=0}^{M-1} c_{n m} \psi_{n m}\right|^{2} w_{n}(t) \mathrm{d} t\right)^{\frac{1}{2}}$. 
Proof Since

$\sigma_{k, M}^{2}=\int_{0}^{1}\left|f(t)-\sum_{n=1}^{2^{k-1}} \sum_{m=0}^{M-1} c_{n m} \psi_{n m}(t)\right|^{2} w_{n}(t) \mathrm{d} t$.

From this Eq. (66), we observe that

$$
\begin{aligned}
& \sigma_{k, M}^{2}=\int_{0}^{1}\left|\sum_{n=2^{k-1}+1}^{\infty} \sum_{m=M}^{\infty} c_{n m} \psi_{n m}(t)\right|^{2} w_{n}(t) \mathrm{d} t \\
& =\sum_{n=2^{k-1}+1}^{\infty} \sum_{m=M}^{\infty} \int_{0}^{1}\left|c_{n m} \psi_{n m}(t)\right|^{2} w_{n}(t) \mathrm{d} t \\
& =\sum_{n=2^{k-1}+1}^{\infty} \sum_{m=M}^{\infty}\left|c_{n m}\right|^{2} \int_{0}^{1}\left|\psi_{n m}(t)\right|^{2} w_{n}(t) \mathrm{d} t .
\end{aligned}
$$

From the orthonormality property of $\psi_{n m}(t)$, we get

$$
\sigma_{k, M}^{2}=\sum_{n=2^{k-1}+1}^{\infty} \sum_{m=M}^{\infty}\left|c_{n m}\right|^{2} .
$$

Using the findings from Eq. (58)

$\sigma_{k, M}^{2}<\frac{\pi \mathcal{K}^{2}}{2^{4}} \sum_{n=2^{k-1}+1}^{\infty} \sum_{m=M}^{\infty} \frac{1}{n^{5}\left(m^{2}-1\right)^{2}}$

Hence

$\sigma_{k, M}<\frac{\sqrt{\pi} \mathcal{K}}{2^{2}}\left(\sum_{n=2^{k-1}+1}^{\infty} \sum_{m=M}^{\infty} \frac{1}{n^{5}\left(m^{2}-1\right)^{2}}\right)^{\frac{1}{2}}$

which completes the proof.

\section{Chebyshev wavelet operational matrix of integration}

The derivation of the integral of the first kind Chebyshev wavelets is presented in this section. This integrals constitute the operational matrix of integration which play a major role in dealing with the problem of the flow equations. For $M=3$ and $k=2$, the six Chebyshev basis functions $\psi_{1,0}(t)=\frac{2}{\sqrt{\pi}}$,

$\psi_{1,1}(t)=\sqrt{\frac{8}{\pi}}(4 t-1)$

$\psi_{1,2}(t)=\sqrt{\frac{8}{\pi}}\left(32 t^{2}-16 t+1\right)$,

on the interval $0 \leq t<\frac{1}{2}$ and

$\psi_{2,0}(t)=\frac{2}{\sqrt{\pi}}$,

$\psi_{2,1}(t)=\sqrt{\frac{8}{\pi}}(4 t-3)$,

$\psi_{2,2}(t)=\sqrt{\frac{8}{\pi}}\left(32 t^{2}-48 t+17\right)$,

on the interval $\frac{1}{2} \leq t \leq 1$, can be integrated from 0 to $t$ giving rise to the following results:

$$
\begin{aligned}
& \int_{0}^{t} \psi_{1,0}(t) \mathrm{d} t= \begin{cases}\frac{2}{\sqrt{\pi}} t, & 0 \leq t \leq \frac{1}{2} \\
\frac{1}{\sqrt{\pi}}, & \frac{1}{2} \leq t \leq 1\end{cases} \\
& =\left[\begin{array}{llllll}
\frac{1}{4} & \frac{1}{4 \sqrt{2}} & 0 & \frac{1}{2} & 0 & 0
\end{array}\right] \Psi(t), \\
& \int_{0}^{t} \psi_{1,1}(t) \mathrm{d} t= \begin{cases}\sqrt{\frac{8}{\pi}}\left(2 t^{2}-t\right), & 0 \leq t \leq \frac{1}{2} \\
0, & \frac{1}{2} \leq t \leq 1\end{cases} \\
& =\left[\begin{array}{llllll}
-\frac{1}{8 \sqrt{2}} & 0 & \frac{1}{16} & 0 & 0 & 0
\end{array}\right] \Psi(t), \\
& \int_{0}^{t} \psi_{1,2}(t) \mathrm{d} t= \begin{cases}\sqrt{\frac{8}{\pi}}\left(\frac{32}{3} t^{3}-8 t^{2}+t\right), & 0 \leq t \leq \frac{1}{2} \\
-\frac{1}{6} \sqrt{\frac{8}{\pi}}, & \frac{1}{2} \leq t \leq 1\end{cases} \\
& =\left[\begin{array}{llllll}
-\frac{1}{6 \sqrt{2}} & -\frac{1}{8} & 0 & -\frac{1}{3 \sqrt{2}} & 0 & 0
\end{array}\right] \Psi(t) \text {, } \\
& \int_{0}^{t} \psi_{2,0}(t) \mathrm{d} t= \begin{cases}0, & 0 \leq t \leq \frac{1}{2} \\
\frac{2}{\sqrt{\pi}} t-\frac{1}{\sqrt{\pi}}, & \frac{1}{2} \leq t \leq 1\end{cases} \\
& =\left[\begin{array}{llllll}
0 & 0 & 0 & \frac{1}{4} & \frac{1}{4 \sqrt{2}} & 0
\end{array}\right] \Psi(t) \text {, } \\
& \int_{0}^{t} \psi_{2,1}(t) \mathrm{d} t= \begin{cases}0, & 0 \leq t \leq \frac{1}{2} \\
\sqrt{\frac{8}{\pi}}\left(2 t^{2}-3 t+1\right), & \frac{1}{2} \leq t \leq 1\end{cases} \\
& =\left[\begin{array}{llllll}
0 & 0 & 0 & -\frac{1}{8 \sqrt{2}} & 0 & \frac{1}{16}
\end{array}\right] \Psi(t) \text {, } \\
& \int_{0}^{t} \psi_{2,2}(t) \mathrm{d} t= \begin{cases}0, & 0 \leq t \leq \frac{1}{2} \\
\sqrt{\frac{8}{\pi}}\left(\frac{32}{3} t^{3}-24 t^{2}+17 t-\frac{23}{6}\right), & \frac{1}{2} \leq t \leq 1\end{cases} \\
& =\left[\begin{array}{llllll}
0 & 0 & 0 & -\frac{1}{6 \sqrt{2}} & -\frac{1}{8} & 0
\end{array}\right] \Psi(t) \text {. }
\end{aligned}
$$


Thus

$\int_{0}^{t} \Psi_{6}(s) \mathrm{d} s=\mathcal{P}_{6 \times 6} \Psi_{6}(t)$,

where

$$
\mathcal{P}_{6 \times 6}=\frac{1}{4}\left[\begin{array}{cccccc}
1 & \frac{1}{\sqrt{2}} & 0 & 2 & 0 & 0 \\
-\frac{1}{2 \sqrt{2}} & 0 & \frac{1}{4} & 0 & 0 & 0 \\
-\frac{2}{3 \sqrt{2}} & -\frac{1}{2} & 0 & -\frac{4}{3 \sqrt{2}} & 0 & 0 \\
0 & 0 & 0 & 1 & \frac{1}{\sqrt{2}} & 0 \\
0 & 0 & 0 & -\frac{1}{2 \sqrt{2}} & 0 & \frac{1}{4} \\
0 & 0 & 0 & -\frac{2}{3 \sqrt{2}} & -\frac{1}{2} & 0
\end{array}\right] \text {. }
$$

The operational matrix $\mathcal{P}_{6 \times 6}$ can be seen as a block matrix given as

$\mathcal{P}_{6 \times 6}=\frac{1}{4}\left(\begin{array}{cc}L_{3 \times 3} & F_{3 \times 3} \\ 0_{3 \times 3} & L_{3 \times 3}\end{array}\right)$

In general, $\mathcal{P}$ is a $2^{k-1} M \times 2^{k-1} M$ given by

$$
\mathcal{P}=\frac{1}{2^{k}}\left(\begin{array}{ccccc}
L & F & F & \cdots & F \\
0 & L & F & \cdots & F \\
\vdots & 0 & \ddots & \ddots & 0 \\
\vdots & \vdots & \vdots & \ddots & F \\
0 & 0 & \cdots & 0 & L
\end{array}\right) .
$$

\section{Chebyshev wavelet formulation of two-phase flow model}

In this section, the Chebyshev wavelet method together with the operational matrix of integration is used to represent the two-phase flow equations. The wavelet representation of the pressure function, $P(x, t)$, is formulated from the pressure equation by letting

$\nabla \cdot \nabla P_{\mathrm{o}}(x, t)=\Psi^{\mathrm{T}}(x) C \Psi(t)$.

The relation is integrated twice with respect to $x$ for $P_{\mathrm{o}}(x, t)$ :

$\int_{0}^{x} \int_{0}^{x} \nabla \cdot \nabla P_{\mathrm{o}}(\tau, t) d \tau d \tau=\int_{0}^{x} \int_{0}^{x} \Psi^{\mathrm{T}}(\tau) C \Psi(t) d \tau d \tau$.

Evaluating the above integral, the operational matrix of integration is used to evaluate the expression on the right, which is given as

$\int_{0}^{x} \nabla P_{\mathrm{o}}(\tau, t)-\nabla P_{\mathrm{o}}(0, t) d \tau=\Psi^{\mathrm{T}}(x)\left(\mathcal{P}^{2}\right)^{\mathrm{T}} C \Psi(t)$.
$P_{\mathrm{o}}(x, t)-P_{\mathrm{o}}(0, t)-x \nabla P_{\mathrm{o}}(0, t)=\Psi^{\mathrm{T}}(x)\left(\mathcal{P}^{2}\right)^{\mathrm{T}} C \Psi(t)$.

Let $x=1$, then based on the boundary conditions governing the flow we obtain an expression for $\nabla P_{\mathrm{o}}(0, t)$ as

$\nabla P_{\mathrm{o}}(0, t)=P_{\mathrm{o}}(1, t)-P_{\mathrm{o}}(0, t)-\Psi^{\mathrm{T}}(1)\left(\mathcal{P}^{2}\right)^{\mathrm{T}} C \Psi(t)$.

Hence, the wavelet formulation of the pressure function for the saturation of oil phase in the reservoir is given as

$$
\begin{aligned}
P_{\mathrm{o}}(x, t)= & \Psi^{\mathrm{T}}(x)\left(\mathcal{P}^{2}\right)^{\mathrm{T}} C \Psi(t)-x \Psi^{\mathrm{T}}(1)\left(\mathcal{P}^{2}\right)^{\mathrm{T}} C \Psi(t) \\
& +(1-x) P_{\mathrm{o}}(0, t)+x P_{\mathrm{o}}(1, t) .
\end{aligned}
$$

From the saturation equation, we let

$\frac{\partial S_{\mathrm{w}}}{\partial t}=\Psi(x) D \Psi(t)$

Equation (85) is integrated once with respect to $t$ to obtain the wavelet formulation of water saturation in the medium:

$\int_{0}^{t} \frac{\partial S_{\mathrm{w}}}{\partial \tau} \mathrm{d} \tau=\int_{0}^{t} \Psi(x) D \Psi(\tau) \mathrm{d} \tau$.

Employing the operational matrix of integration, the integral evaluates to

$S_{\mathrm{w}}(x, t)=S_{\mathrm{w}}(x, 0)+\Psi(x) D \mathcal{P} \Psi(t)$.

The known functions $q_{\mathrm{w}}, q_{\mathrm{o}}, P_{\mathrm{c}}$, and $Z$ are also decomposed with the Chebyshev wavelets basis as

$q_{\mathrm{w}}=\Psi^{\mathrm{T}}(x) A \Psi(t)$,

$q_{\mathrm{o}}=\Psi^{\mathrm{T}}(x) B \Psi(t)$,

$\nabla \cdot \nabla P_{\mathrm{c}}=\Psi^{\mathrm{T}}(x) E \Psi(t)$,

$\nabla \cdot \nabla Z=\Psi^{\mathrm{T}}(x) H \Psi(t)$.

Note that $\langle\Psi(x),\langle * *, \Psi(t)\rangle\rangle$ is used to evaluate the wavelet coefficients, and $* *$ in the inner product accepts the various functions as input to evaluate the respective wavelet coefficients. Substituting the wavelet representation of the various functions into Eqs. (10) and (11), the wavelet formulation of the two-phase flow model was obtained as

$$
\begin{aligned}
- & K\left(\lambda_{\mathrm{w}}+\lambda_{\mathrm{o}}\right) \Psi(x) C \Psi(t) \\
& -K g\left(\lambda_{\mathrm{w}} \rho_{\mathrm{w}}+\lambda_{\mathrm{o}} \rho_{\mathrm{o}}\right) \Psi(x) H \Psi(t) \\
& +K \lambda_{\mathrm{w}} \Psi(x) E \Psi(t)=\Psi(x) A \Psi(t)+\Psi(x) B \Psi(t)
\end{aligned}
$$

$\phi \Psi(x) D \Psi(t)+K \lambda_{\mathrm{w}} \Psi(x) C \Psi(t)=\Psi^{\mathrm{T}}(x) A \Psi(t)$.

Since $A, B, E$, and $H$ are known, the two equations are solved simultaneously for $C$ and $D$. The solution to $P_{\mathrm{o}}(x, t)$ and $S_{\mathrm{w}}(x, t)$ can be reconstructed from their wavelet coefficients $C$ and $D$, respectively, using Eqs. (84) and (87).

This results to 
Table 1 Relevant data for the oil-water reservoir simulation

\begin{tabular}{ll}
\hline Relevant data & \\
\hline Porosity & $\phi=0.2$ \\
Absolute permeability & $k=1$ \\
Water viscosity & $\mu_{\mathrm{w}}=1 \mathrm{cP}$ \\
Oil viscosity & $\mu_{\mathrm{o}}=0.25 \mathrm{cP}$ \\
Density of water & $\rho_{\mathrm{w}}=1000 \mathrm{~kg} / \mathrm{m}^{3}$ \\
Density of oil & $\rho_{\mathrm{o}}=1000 \mathrm{~kg} / \mathrm{m}^{3}$ \\
Residual saturations & $S_{\mathrm{rw}}=S_{\mathrm{ro}}=0 / S_{\mathrm{rw}}=0, S_{\mathrm{ro}}=0.2$ \\
\hline
\end{tabular}

\section{Numerical results}

The study considered a reservoir saturated with two completely immiscible fluid phases: water and oil. The velocity of the flow is estimated based on the extended Darcy's law which considers the effect of gravitational force on the process. Relevant data for the simulation of the formulated problem is presented in Table 1.

In this section, the results for the numerical simulation of flow equations formulated based on the proposed Chebyshev wavelet method is presented. Different test cases were considered for flow of fluids in the reservoir based on different treatment of the capillary pressure, $\left(P_{\mathrm{c}}\right)$. The capillary function (Hoteit and Firoozabadi 2008a)

$P_{\mathrm{c}}\left(S_{\mathrm{w}}\right)=-\frac{B_{\mathrm{c}}}{\sqrt{k}} \log \left(S_{\mathrm{e}}\right)$

was adapted for the study taking $B_{\mathrm{c}}$ which is a positive parameter to be 25 as explained by Kou and Sun (2010) and $S_{\mathrm{e}}$ is the normalized saturation given as

$S_{\mathrm{e}}=\frac{S_{\mathrm{w}}-S_{\mathrm{rw}}}{1-S_{\mathrm{rw}}-S_{\mathrm{ro}}}$,

where $S_{\text {ro }}$ and $S_{\text {rw }}$ are the residual saturations of oil and water, respectively. Figure 1 shows correlation between the capillary pressure and the wetting phase, indicating a decrease in capillary pressure with increase in the saturation of wetting phase in the reservoir.

The function employed for the evaluation of the relative permeabilities for both fluid phases is

$k_{\mathrm{rw}}=S_{\mathrm{e}}^{\alpha}$,

$k_{\text {ro }}=\left(1-S_{\mathrm{e}}\right)^{\alpha}$,

and $\alpha$ is a positive integer. Simulation for the study was carried out for $\alpha=4$. The effect of the residual saturations was also taken into account for the fluid phases. For an oil-water system, flow of the oil is possible when the fluid phase saturation is greater than the residual oil saturation. In one instance, both the residual saturations of the fluid

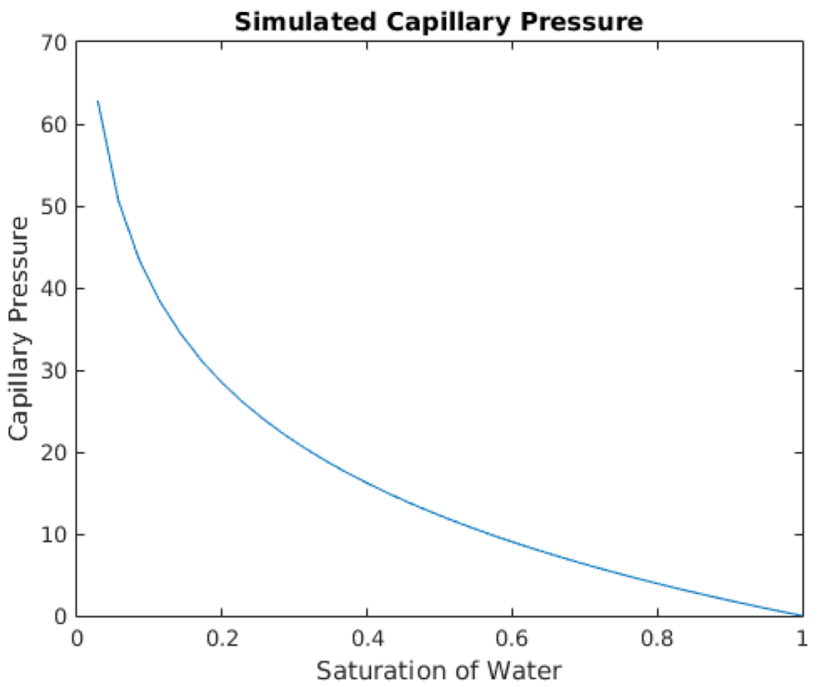

Fig. 1 Correlation between capillary pressure and saturation of water

phases were considered to be zero (Kou and Sun 2010). The other instance considered the residual saturation of the oil $S_{\text {ro }}=0.2$, Hoteit and Firoozabadi (2008a), as presented in Table 1.

Figure 2 shows the distribution of the saturation for the oil-water reservoir system limited by the saturation constraint of the flow. The saturation of the water is noted to increase with time during the production process of the oil, while the saturation of the oil decreases. Results are in normal units of both temporal and spatial variable. Some discontinuity as observed from the results and according to Kou and Sun (2010) may be due to the continuity in the treatment of the capillary pressure. Corresponding profile showing the distribution of the oil and water in the reservoir is presented in Fig. 3. The saturations profiles in all the cases obey the constraint in the saturation (Eq. (3)).

The capillary pressure is responsible for the difference in the pressure of the two fluids. According to Eq. (4), neglecting the capillary pressure results to same pressure in both fluid phases. The evidence of this is shown in Fig. 4. This pressure of the fluids flowing in the reservoir is observed to decrease with time but higher with increase in the depth of the reservoir. The pressure dynamics in the fluids follow similar pattern for all instances with difference in pressure due to capillary treatment.

When gravity is ignored, the saturation of the oil rather increased, whereas that of the water decreased with time, as shown in Fig. 5. The distribution of the two fluids for this scenario is presented in Fig. 6. However, the dynamics changes with the inclusion of the capillary pressure, as shown in Fig. 7. In all the cases discussed so far, the residual saturation had been neglected. The saturation profile is presented in Fig. 8 for the two-phase flow with the residual 

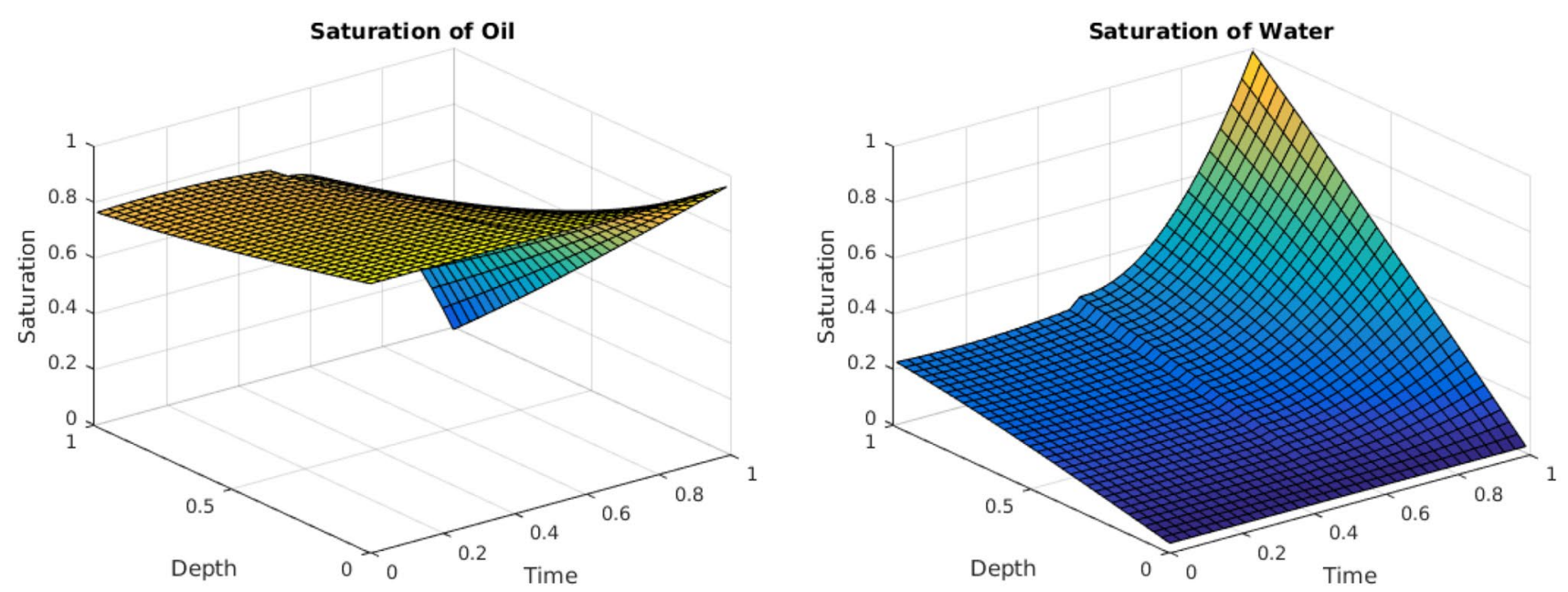

Fig. 2 Saturation of fluids in the reservoir when the residual saturations are neglected

Fig. 3 Distribution of fluids in the reservoir with neglected residual saturations
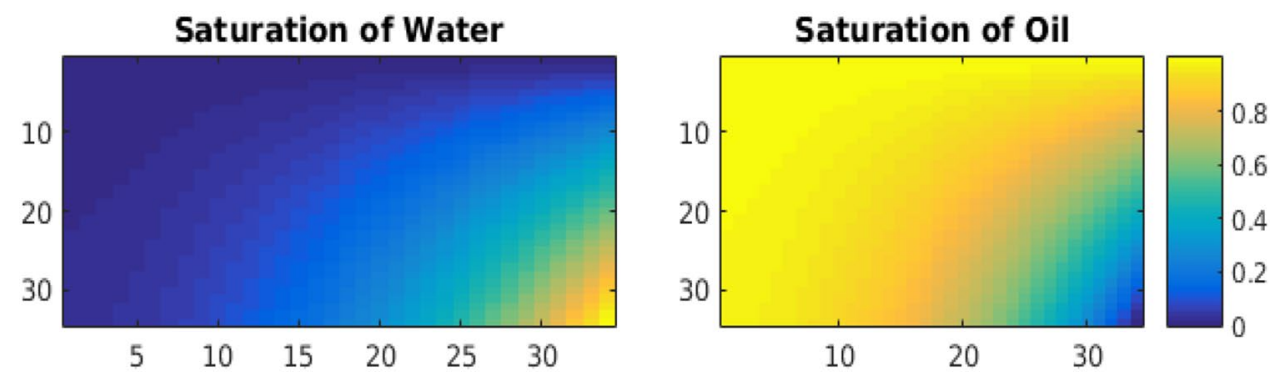

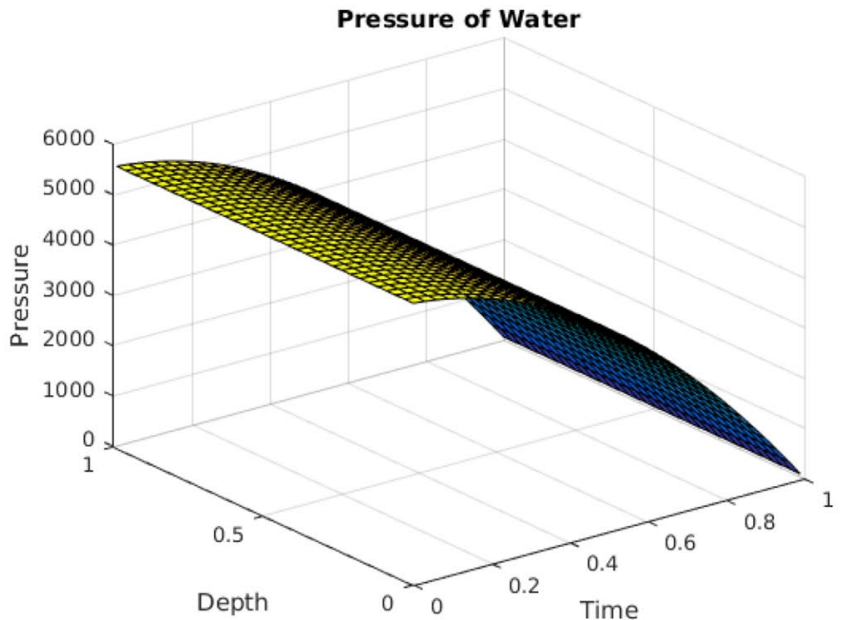

Fig. 4 Pressure distribution in the reservoir

saturation of the oil taken to be $S_{\text {ro }}=0.2$. As is observed, capillary pressure has a great influence on the flow pattern of the fluids in the reservoir.

In Fig. 9, the density of the wetting phase was kept at $1000 \mathrm{~kg} / \mathrm{m}^{3}$ as in the previous cases, but that of the nonwetting phase is $660 \mathrm{~kg} / \mathrm{m}^{3}$. All the other parameter values are maintained including the force of gravity. Unlike the earlier results, where the distribution of the saturation had some discontinuity, varying the density of the fluids completely eliminates the discontinuity. Interestingly, the dynamics of the fluid dispersion in the medium is maintained. 

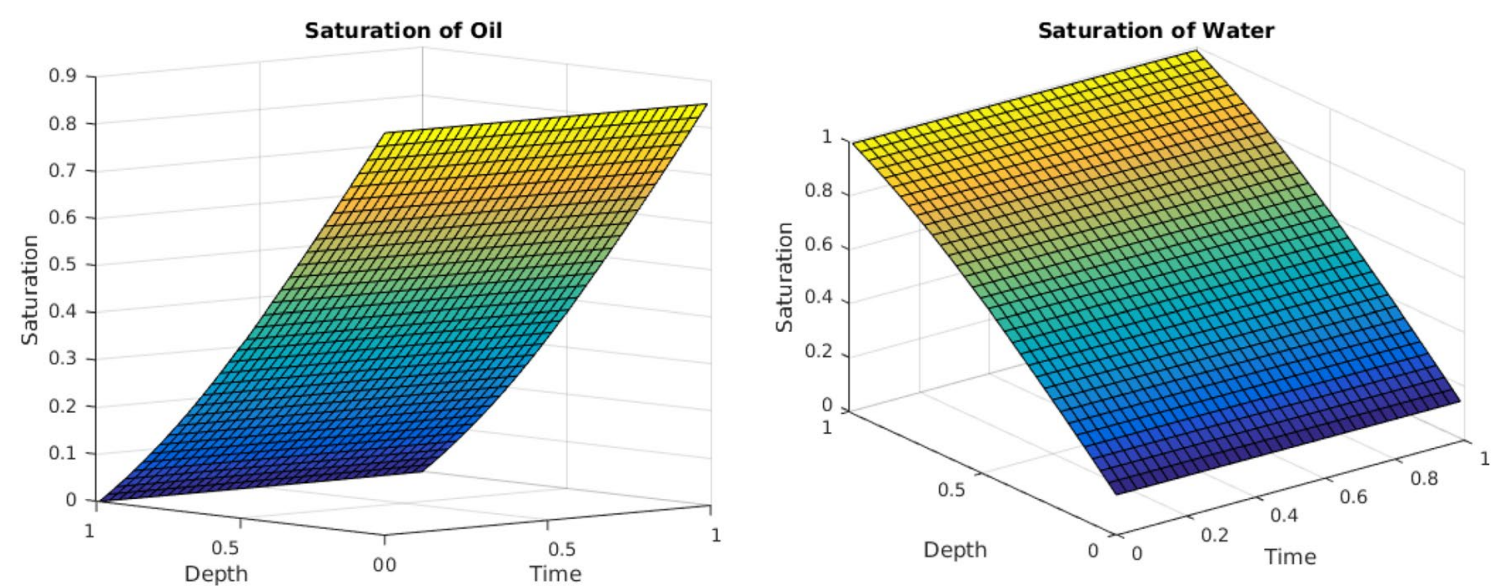

Fig. 5 Fluid saturation in the reservoir in the absence of gravity

Fig. 6 Saturation profile in the reservoir considering capillary pressure in the absence of gravity
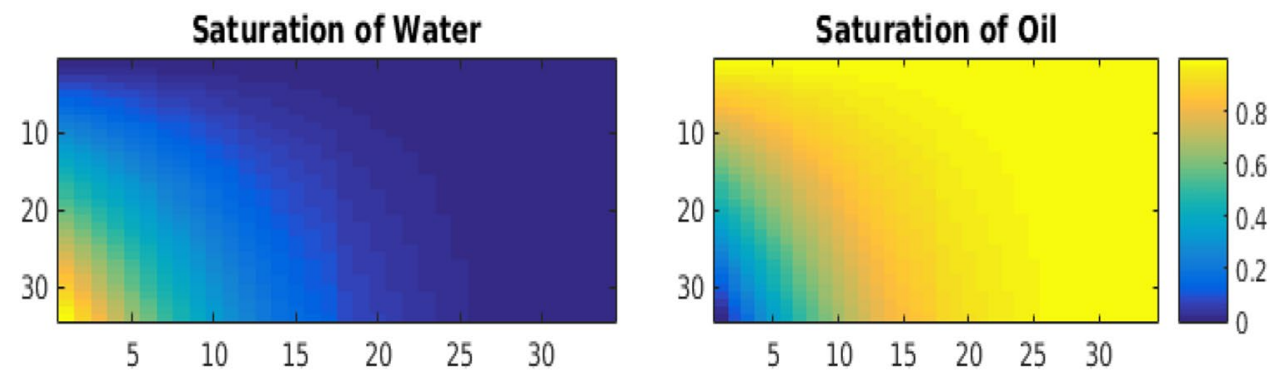

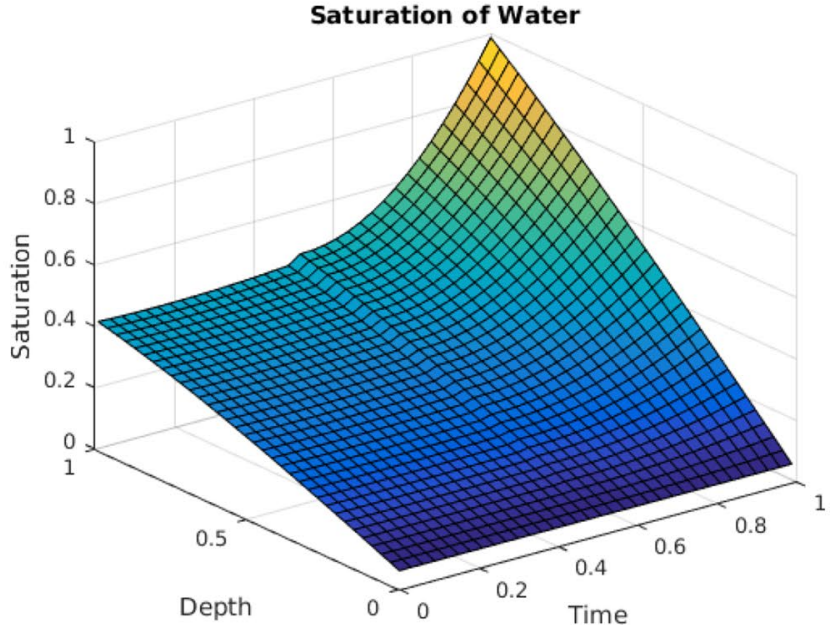

Fig. 7 Fluid saturation in the reservoir with capillary pressure in the presence of gravity

\section{Conclusions}

The proposed Chebyshev wavelets method has been successfully implemented in studying the two-phase slightly compressible flow in combination with their operational matrix of integration. This method was developed based on the first kind Chebyshev polynomial. The corresponding algorithm converges quite well, stable, and adapts to changing dynamics. Key to this study is the inclusion and treatment of the capillary pressure. The fluid dispersion in the reservoir displayed some discontinuities which in the literature may be due to the continuity of the capillary treatment. The study also revealed that the other properties 
Fig. 8 Saturation profile in the reservoir with the non-wetting phase residual saturation $S_{\text {ro }}=0.2$
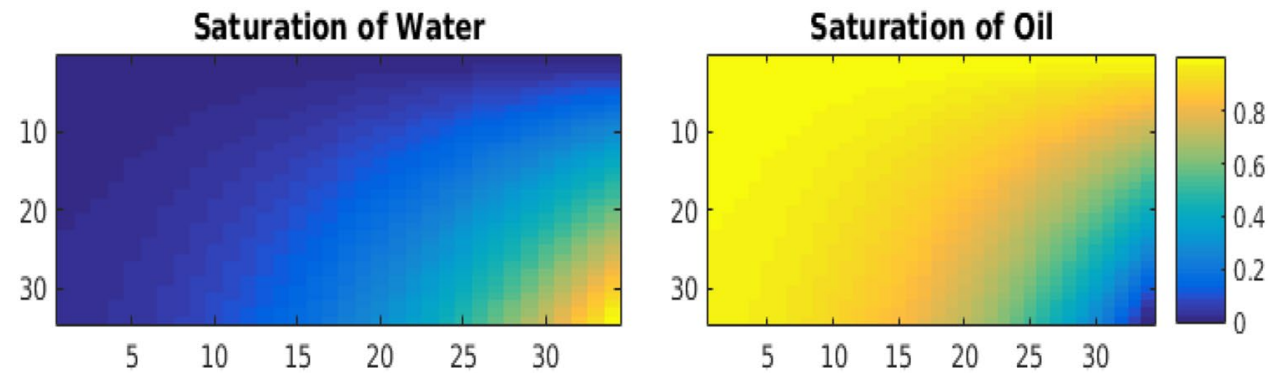
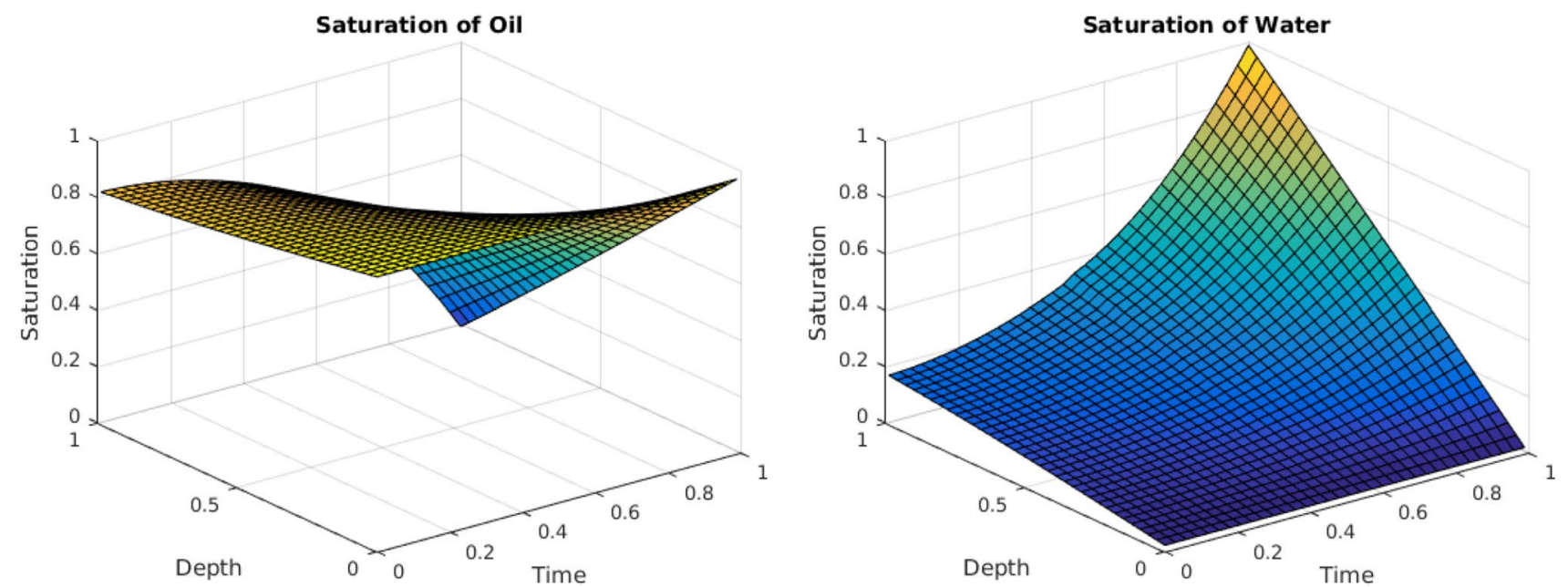

Fig. 9 Fluid saturation in the reservoir with different values for density of the two fluid phases

of the process such as density could completely eliminate the discontinuity in saturation of the fluids.

Acknowledgements We will like to acknowledge the support received from the National Institute for Mathematical Sciences, Ghana for this study

\section{Compliance with ethical standards}

Competing interests The authors declare that they have no competing interests.

Open Access This article is distributed under the terms of the Creative Commons Attribution 4.0 International License (http://creativeco mmons.org/licenses/by/4.0/), which permits unrestricted use, distribution, and reproduction in any medium, provided you give appropriate credit to the original author(s) and the source, provide a link to the Creative Commons license, and indicate if changes were made.

\section{References}

Adibi H, Assari P (2010) Chebyshev wavelet method for numerical solution of Fredholm integral equations of the first kind. Math Probl Eng. https://doi.org/10.1155/2010/138408
Ahammad MJ, Alam JM (2017) A numerical study of two-phase miscible flow through porous media with a lagrangian model. J Comput Multiph Flows 9(3):127-143. https://doi.org/10.1177/17574 82X17701791

Alam JM (2017) A wavelet based numerical simulation technique for two-phase flows using the phase field method. Comput Fluids 146:143-153

Al-Rbeawi S (2017) Analytical models \& type-curve matching techniques for reservoir characterization using wellbore storage dominated flow regime. J Pet 4(2):223-229. https://doi.org/10.1016/j. petlm.2017.09.001

Amaziane B, Jurak M (2007) A new formulation of immiscible compressible two-phase flow in porous media. Elsevier Science, Amsterdam

Amoako-Yirenkyi P, Awashie GE, Dontwi IK (2016) On the analysis and numerical formulation of miscible fluid flow in porous media using Chebyshev wavelets collocation method. J Appl Math Phys 4:1210-1221

Araghi MAF, Daliri S, Bahmanpour M (2012) Numerical solution of integro-differential equation by using Chebyshev wavelet operational matrix of integration. Int J Math Model Comput 02(02):127-136

Begum N (2009) Reservoir parameter estimation for reservoir simulation using ensemble Kalman filter. Nav Res Logist Q

Daubechies I (1992) Ten lectures on wavelets. Society for Industrial and Applied Mathematics, Philadelphia 
de la Cruz LM, Monsivais D (2013) Parallel numerical simulation of two-phase flow model in porous media using distributed and shared memory architectures. Geofis Int 53(1):59-75

El-Amin M, Meftah R, Salama A, Sun S (2015) Numerical treatment of two-phase flow in porous media including specific interfacial area. In: Elsevier ICCS 2015 international conference on computational science, vol 51, pp 1249-1258

Epshteyn Y, Riviere B (2006) On the solution of incompressible two-phase flow by a p-version discontinuous Galerkin method. Commun Numer Methods Eng 22(7):741-751

Ewing RE (1983) The mathematics of reservoir simulation. Society of Applied Mathematics, Laramie

Foroozesh J, Barzegari D, Ayatollahi S, Jahanmiri A (2008) Simulation of water coning in oil reservoir using a corrected IMPES method. Iran J Chem Eng 5(4):3-11

Heydari MH, Hooshmandasl MR, Maalek Ghaini FM, Mohammadi F (2012) Wavelet collocation method for solving multiorder fractional differential equations. J Appl Math 2012:542401. https:// doi.org/10.1155/2012/542401

Heydari M, Hooshmandasl M, Ghaini RM (2014) A new approach of Chebyshev wavelets method for partial differential equations with boundary conditions of the telegraph type. Appl Math Model $1597-1606$

Hooshmandasl MR, Heydari MH, Ghaini FMM (2012) Numerical solution of the one-dimensional heat equation by using Chebyshev wavelets method. Appl Comput Math 1:2168-9679

Hoteit H, Firoozabadi A (2008a) Numerical modeling of two-phase flow in heterogeneous permeable media with different capillarity pressures. Adv Water Resour 31(1):56-73

Hoteit H, Firoozabadi A (2008b) An efficient numerical model for incompressible two-phase flow in fractured media. Adv Water Resour 31(6):891-905

Kou J, Sun S (2010) A new treatment of capillarity to improve the stability of IMPES two-phase flow formulation. Comput Fluids 39(10):1923-1931
Lepik U (2011) Solving PDEs with the aid of two-dimensional haar wavelets. Comput Math Appl 61:1873-1879

Li Y (2010) Solving a nonlinear fractional differential equation using Chebyshev wavelets. Commun Nonlinear Sci Numer Simul 15(9):2284-2292

Mozolevski I, Schuh L (2013) Numerical simulation of two-phase immiscible incompressible flows in heterogeneous porous media with capillary barriers. J Comput Appl Math 242:12-27

Pasquier S, Quintard M, Davit Y (2017) Modeling two-phase flow of immiscible fluids in porous media: Buckley-Leverett theory with explicit coupling terms. Phys Rev Fluids 2:104101. https://doi. org/10.1103/PhysRevFluids.2.104101

Peaceman DW (1977) Fundamentals of numerical reservoir simulation. Elsevier Scientific Publishing Company, Amsterdam

Soulaine C, Davit Y, Quintard M (2013) A two-pressure model for slightly compressible single phase flow in bi-structured porous media. Chem Eng Sci 96:55-70

Sun T, Yuan Y (2015) Mixed finite element method and the characteristics-mixed finite element method for a slightly compressible miscible displacement problem in porous media. Math Comput Simul 107:24-45

Szymkiewicz A (2007) Numerical simulation of one-dimensional two-phase flow in porous media. Arch Hydro Eng Environ Mech 54(2):117-136

Yuan Y, Cheng A, Yang D, Li C (2015) Theory and application of fractional step characteristic finite difference method in numerical simulation of second order enhanced oil production. Acta Math Sci 35(6): 1547-1565

Zhong W, Li C, Kou J (2013) Numerical fractional-calculus model for two-phase flow in fractured media. Adv Math Phys 2013:429835. https://doi.org/10.1155/2013/429835

Publisher's Note Springer Nature remains neutral with regard to jurisdictional claims in published maps and institutional affiliations. 